\title{
Fenologia e produção das videiras ‘Alicante’ e ‘Syrah’ em safra fora de época
}

\author{
Phenology and production of 'Alicante' and 'Syrah' grapevines grown out of season
}

\author{
Alessandro Jefferson Sato ${ }^{\mathrm{I}}$ Adriane Marinho de Assis ${ }^{\mathrm{II}}$ Lilian Yukari Yamamoto ${ }^{\mathrm{III}}$ \\ Lidiane Carla Villanova Miotto ${ }^{\mathrm{III}}$ Edmar Clemente $^{\mathrm{IV}}$ Sergio Ruffo Roberto $^{\mathrm{V}}$
}

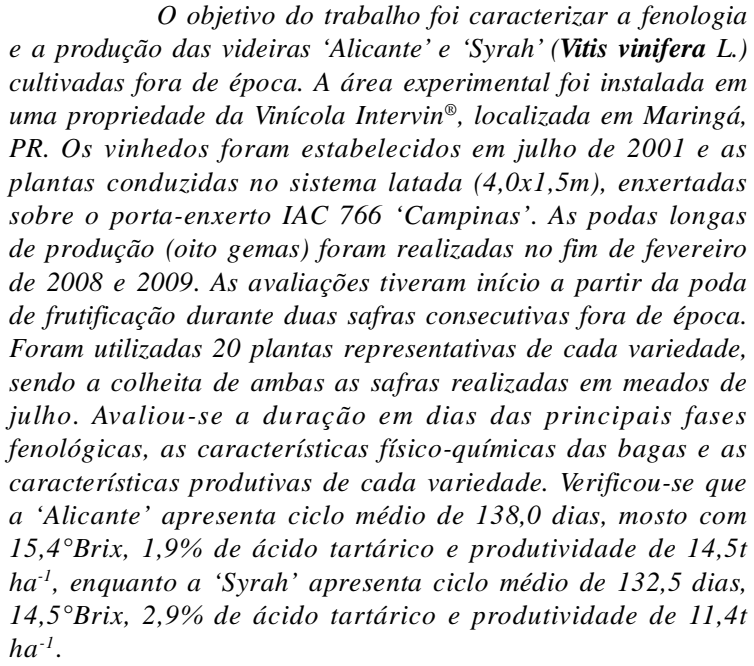
RESUMO
\end{abstract}

Palavras-chave: $u v a$, sólidos solúveis totais, poda.

\section{ABSTRACT}

This study aimed to characterize the phenology and the production of 'Alicante' and 'Syrah' grapes (Vitis vinifera L.) produced out of season. The experimental area was located in a property belonging to the Intervin ${ }^{\circledR}$ Winery, at Maringá, PR. The vineyards were established in July, 2001 and the vines were trained in an overhead trellising system (4.0x1.5m), grafted on IAC 766 'Campinas' rootstock. The long pruning of production (eight buds) was held in late February of 2008 and 2009. The evaluations started from the pruning of production, in two years, using 20 representative vines of each variety, and the harvesting of both crops were performed on early July. The phenology of the trees was evaluated as the duration in days of the most important phases, as well it was analyzed the berries physico-chemical characteristics. The production characteristics of each variety were also estimated. It was verified that 'Alicante' showed a mean cycle of 138.0 days, must with $15.4^{\circ}$ Brix, $1.9 \%$ of tartaric acid and productivity of $14.5 \mathrm{t} \mathrm{ha}^{-1}$, while 'Syrah' showed a mean cycle of 132.5 days, $14.5^{\circ}$ Brix, $2.9 \%$ of tartaric acid and productivity of $11.4 \mathrm{t} \mathrm{ha}$.

Key words: grapes, total soluble solids, pruning.

$\mathrm{Na}$ cadeia produtiva de uvas e vinhos, busca-se cada vez mais elevar a qualidade dos produtos. Para tanto, diversas iniciativas têm sido tomadas com o propósito de se obter melhores índices de qualidade nas uvas (JUBILEU et al., 2010). Uma das técnicas utilizadas é a produção fora de época que permite a colheita em períodos com baixa precipitação e, dessa forma, evita a ocorrência das podridões típicas de fim de ciclo nas safras regulares (KISHINO \& CARAMORI, 2007).

Dentre as videiras destinadas à vinificação, destaca-se a 'Alicante' e a 'Syrah' (Vitis vinifera L.),

IPrograma Nacional de Pós-doutorado em Agronomia, Universidade Estadual do Centro-Oeste (Unicentro), Guarapuava, PR, Brasil.

"Programa Nacional de Pós-doutorado em Agronomia, Universidade Estadual de Londrina (UEL), Londrina, PR, Brasil. IIIPrograma de Pós-graduação em Agronomia, UEL, Londrina, PR, Brasil.

${ }^{\mathrm{IV}}$ Departamento de Química, Universidade Estadual de Maringá (UEM), Maringá, PR, Brasil.

`Departamento de Agronomia, UEL, CP 6001, 86051-990, Londrina, PR, Brasil. E-mail: sroberto@uel.br. Autor para correspondência. 
ambas de origem francesa, que são cultivadas em diversas regiões do mundo (LEÃO et al., 2009). O comportamento fenológico e produtivo dessas videiras em safras regulares são bem conhecidos, como se pode observar nos trabalhos realizados por SOUZA et al. (2002) e FAVERO et al. (2008), ambos no Sul de Minas Gerais, assim como ORLANDO et al. (2008) na região de Jundiaí, SP, entre outros. No entanto, ressalta-se que para a produção de uvas finas, destinadas à vinificação em safra fora de época, sobretudo em regiões de clima subtropical, são raras as informações disponíveis. Portanto, antes de se indicar o uso dessa técnica, é fundamental que se avalie comportamento destas uvas nas regiões e nos períodos em que se deseja implantar a cultura.

Dessa forma, este trabalho teve o objetivo de caracterizar o comportamento fenológico e produtivo das uvas 'Alicante' e 'Syrah' cultivadas em safra fora de época no Norte do Paraná.

O trabalho foi conduzido em vinhedos da 'Alicante' e da 'Syrah' pertencentes à Vinícola Intervin ${ }^{\circledR}$, localizada em Maringá, PR $\left(23^{\circ} 25^{\prime}\right.$ ', $51^{\circ} 57^{\prime} \mathrm{W}$, altitude de 542m). O clima da região é classificado como Cfa. A precipitação média anual é de $1.596 \mathrm{~mm}$ e a tendência é de concentração das chuvas no verão (IAPAR, 2010a). Foram utilizadas 20 plantas representativas de cada variedade, conduzidas em sistema latada, em espaçamento de 4,0x1,5m, e enxertadas em julho de 2001 sobre o porta-enxerto IAC 766 'Campinas'. As Avaliações foram realizadas durante duas safras fora de época consecutivas, em 2008 e 2009. As podas curtas de formação (duas gemas) foram realizadas no fim de agosto de 2007 e 2008 , e as podas longas de frutificação (oito gemas), no fim de fevereiro de 2008 e 2009, os cachos que porventura surgiram foram eliminados.

Para a caracterização da fenologia, foi avaliada, por meio de observações visuais, a duração em dias dos seguintes estádios a partir da poda de frutificação (PO) (BAILLOD \& BAGGIOLINI, 1993): Gema-algodão (GA); Brotação (BR); Aparecimento da inflorescência (AI); Florescimento (FL); Início da maturação(IM); Colheita (CO).

Por ocasião da colheita, foram avaliados o teor de sólidos solúveis totais (SST) e acidez titulável (AT). O teor de SST do mosto foi determinado em refratômetro digital de bancada com compensação de temperatura e o resultado expresso em ${ }^{\circ}$ Brix. A determinação da AT do mosto foi realizada em titulador semi-automático (solução de $\mathrm{NaOH} 0,1 \mathrm{~N}$ e ponto final da titulação: $\mathrm{pH}=8,2)$, e o resultado expresso em \% de ácido tartárico (INSTITUTO ADOLFO LUTZ, 1985). Para a as características produtivas, em função do número e massa de cachos por planta e da densidade de plantas, foram estimadas a produção por planta $(\mathrm{kg})$ e a produtividade $\left(\mathrm{tha}^{-1}\right)$.

Destaca-se que, para ambas as videiras na safra de 2009, o ciclo foi mais longo em relação ao de 2008 (Tabela 1). Por outro lado, verifica-se que o subperíodo entre o IM-CO foi menor em 2009 em relação ao de 2008 , pois, em virtude da elevada precipitação ocorrida durante essa fase na segunda safra $(85 \mathrm{~mm} \mathrm{em}$ 2008 e 176mm em 2009), houve a necessidade de se antecipar a colheita das uvas para evitar possíveis danos nos aspectos qualitativos dos cachos.

Com relação ao teor de SST, a 'Alicante' apresentou em média $15,4^{\circ}$ Brix e a 'Syrah' $14,5^{\circ}$ Brix (Tabela 2). Para a 'Alicante', o teor de SST foi mais

Tabela 1 - Duração do ciclo e das diferentes fases fenológicas das videiras 'Alicante' e 'Syrah' produzidas fora de época, nas safras de 2008 e 2009. Maringá, PR.

\begin{tabular}{|c|c|c|c|c|c|c|c|}
\hline \multirow{2}{*}{ Safras } & \multicolumn{7}{|c|}{ - } \\
\hline & PO-GA & GA-BR & BR-AI & AI-FL & FL-IM & IM-CO & $\mathrm{PO}-\mathrm{CO}$ \\
\hline & \multicolumn{7}{|c|}{ 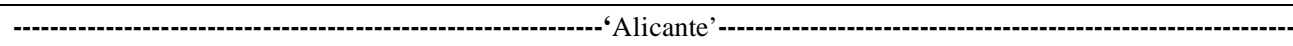 } \\
\hline 2008 & 6 & 4 & 19 & 8 & & 46 & 133 \\
\hline 2009 & 10 & 7 & 14 & 9 & 62 & 41 & 143 \\
\hline Média & 8,0 & 5,5 & 16,5 & 8,5 & 56,0 & 43,5 & 138,0 \\
\hline Desvio & 2,8 & 2,1 & 3,5 & 0,7 & 8,5 & 3,5 & 7,1 \\
\hline 2008 & 8 & 6 & 19 & 6 & 50 & 40 & 129 \\
\hline 2009 & 9 & 7 & 13 & 8 & 62 & 37 & 136 \\
\hline Média & 8,5 & 6,5 & 16,0 & 7,0 & 56,0 & 38,5 & 132,5 \\
\hline Desvio & 0,7 & 0,7 & 4,2 & 1,4 & 8,5 & 2,1 & 4,9 \\
\hline
\end{tabular}

PO-GA: poda-gema-algodão; GA-BR: gema-algodão-brotação; BR-AI: brotação-aparecimento da inflorescência; AI-FL: aparecimento da inflorescência-florescimento; FL-IM: florescimento-início da maturação; IM-CO: início da maturação-colheita; PO-CO: poda-colheita. 
Tabela 2 - Características físico-químicas e produtivas das videiras 'Alicante' e 'Syrah’ produzidas fora de época, nas safras de 2008 e 2009. Maringá, PR.

\begin{tabular}{|c|c|c|c|}
\hline \multirow{2}{*}{ Variáveis } & \multicolumn{3}{|c|}{ 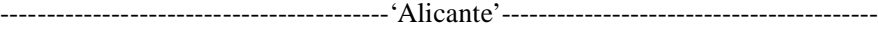 } \\
\hline & Safra 2008 & Safra 2009 & Média \\
\hline $\mathrm{SST}^{\mathrm{a} /}\left({ }^{\circ}\right.$ Brix $)$ & $16,4 \pm 0,3$ & $14,5 \pm 0,2$ & $15,4 \pm 1,3$ \\
\hline $\mathrm{AT}^{\mathrm{b} /}(\%$ de ácido tartárico $)$ & $1,3 \pm 0,09$ & $2,6 \pm 0,01$ & $1,9 \pm 0,4$ \\
\hline Produção por planta ${ }^{\mathrm{cl}}(\mathrm{kg})$ & $11,0 \pm 1,7$ & $6,4 \pm 1,1$ & $8,7 \pm 3,2$ \\
\hline Produtividade $^{\mathrm{d} /}\left(\mathrm{t} \mathrm{ha}^{-1}\right)$ & $18,4 \pm 2,9$ & $10,7 \pm 1,9$ & $14,5 \pm 5,4$ \\
\hline $\mathrm{SST}^{\mathrm{a} /}\left({ }^{\circ} \mathrm{Brix}\right)$ & $14,8 \pm 0,4$ & $\begin{array}{c}--' S y r a h '-- \\
14,3 \pm 0,2\end{array}$ & $14,5 \pm 0,4$ \\
\hline $\mathrm{AT}^{\mathrm{b} /}$ (\% de ácido tartárico) & $2,8 \pm 0,05$ & $2,0 \pm 0,01$ & $2,4 \pm 0,7$ \\
\hline Produção por planta ${ }^{\mathrm{cl}}(\mathrm{kg})$ & $5,6 \pm 0,7$ & $8,1 \pm 0,9$ & $6,8 \pm 1,7$ \\
\hline Produtividade $^{\mathrm{d} /}\left(\mathrm{t} \mathrm{ha}^{-1}\right)$ & $9,4 \pm 1,1$ & $13,4 \pm 1,5$ & $11,4 \pm 2,8$ \\
\hline
\end{tabular}

a/: Sólidos solúveis totais.

b/: Acidez titulável.

c/: Estimativa em função do número médio de cachos por planta e da sua massa média.

d/: Estimativa em função da produção média por planta e da densidade por hectare.

elevado na safra de 2008 em relação à de 2009 , ocasionado pelo fato de que na primeira safra a precipitação durante a fase de maturação foi constante e houve a necessidade de antecipar a colheita.

Com base no histórico climático do Norte do Paraná (IAPAR, 2010b), o cultivo de videiras em safra fora de época ocorre em períodos de menor precipitação durante a fase de maturação. No entanto, trata-se de uma região de clima subtropical, que, em alguns anos, pode ocorrer períodos chuvosos durante a safra fora de época, conforme observado na safra de 2009, o que tornou necessário adotar medidas para manter a qualidade das uvas.

Com relação à 'Syrah', observa-se, na tabela 2, que a precipitação não influenciou o teor de SST entre as safras avaliadas. Esse comportamento está relacionado ao fato de que cada cultivar responde de maneira diferente às variações climáticas (CAMARGO et al., 2004). SOUZA et al. (2002) verificaram que a 'Syrah', em a safra regular no Sul de Minas Gerais, apresenta teor de SST de $16,5^{\circ}$ Brix. A diferença em relação ao obtido nesse trabalho está relacionada ao fato de que a fase IM-CO foi em média 13 dias mais curta no Norte do Paraná do que no Sul de Minas Gerais. Além disso, deve-se considerar que este trabalho foi realizado em safra fora de época, ou seja, em período em que a temperatura média do ar é menor, o que influencia na síntese e no acúmulo de açúcar (WINKLER, 1974).

Com base nos teores de SST das uvas avaliadas neste trabalho, considera-se necessário realizar a chaptalização no momento da vinificação, tendo em vista que, pela legislação brasileira (Lei 7.678/
88), os vinhos devem apresentar no mínimo $10^{\circ} \mathrm{GL}$. Em relação a AT (Tabela 2), destaca-se que a 'Alicante' apresentou teor superior ao encontrado por WYLER et al. (2007) no Vale do São Francisco. Essa diferença está relacionada aos aspectos climáticos de cada região, pois, em condições de clima seco e quente, a degradação dos ácidos é maior (BLOUIN \& GUIMBERTEAU, 2004).

Na safra de 2008, a AT da 'Alicante' foi menor em relação a 2009 (Tabela 2), fator este relacionado ao fato de que, na segunda safra, as uvas foram colhidas antecipadamente, em função do seu estado fitossanitário. Para a 'Syrah', observou-se comportamento distinto (Tabela 2), pois, na safra de 2008, a AT foi mais elevada em relação à de 2009. Portanto, observou-se que a precipitação mais elevada na safra de 2009 não causou danos aos aspectos fitossanitários da 'Syrah', e contribuiu para a diluição dos ácidos orgânicos, que ocorre em função do acúmulo de água nas bagas (BLOUIN \& GUIMBERTEAU, 2004). Ressalta-se que os valores de AT observados nesse trabalho (Tabela 2) são satisfatórios, por se tratar de uvas destinadas à vinificação, tendo em vista que, no processo de fermentação, ocorre redução da acidez do mosto (RIZZON et al., 1998).

Com relação aos aspectos produtivos, é recomendável que a produtividade não ultrapasse $12 \mathrm{t}$ $\mathrm{ha}^{-1}$, pois se procura priorizar os aspectos qualitativos em relação aos quantitativos (SOUZA et al., 2002). Neste trabalho, observa-se que as videiras avaliadas apresentaram, em média, produção e produtividade próximas ao desejável para a elaboração de vinhos. No 
entanto, em função das condições climáticas locais, em algumas safras, a produtividade pode ser elevada, sendo necessário realizar o controle da produção (Tabela 2).

Verifica-se que, na produção fora de época das videiras 'Alicante' e 'Syrah', os cachos apresentam ótimo aspecto fitossanitário e duração de ciclo em média de 138,0 e 132,5 dias, respectivamente, sendo que, em relação aos aspectos químicos do mosto, os valores observados são de $15,4^{\circ}$ Brix e $1,9 \%$ de ácido tartárico para a 'Alicante' e de $14,5^{\circ}$ Brix e $2,9 \%$ de ácido tartárico para a 'Syrah', enquanto que a produtividade é de 14,5t $\mathrm{ha}^{-1}$ e $11,4 \mathrm{t} \mathrm{ha}{ }^{-1}$, respectivamente. Dessa forma, considera-se que as uvas obtidas neste trabalho apresentam potencial para serem utilizadas como matéria-prima na elaboração de vinhos finos.

\section{REFERÊNCIAS}

BAILLOD, M.; BAGGIOLINI, M. Lês stades repères de la vigne. Revue Suisse Viticulture Horticulture, Croft, v.25, n.1, p.7-9, 1993.

BLOUIN, J.; GUIMBERTEAU, G. Maduracion y madurez de la uva. Madrid: Mundi-Prensa, 2004. 151p.

CAMARGO, U.A. et al. Introdução e avaliação de novas cultivares para vinho no Vale do São Francisco. In: WORKSHOP INTERNACIONAL DE PESQUISA: A PRODUÇÃO DE VINHOS EM REGIÕES TROPICAIS, 2004, Petrolina. Anais... Petrolina: Embrapa Uva e Vinho, 2004. p.97-101.

FAVERO, A.C. et al. Viabilidade de produção da videira 'Syrah', em ciclo de outono inverno, no Sul de Minas Gerais. Revista Brasileira de Fruticultura, Jaboticabal, v.30, n.3, p.685-690, 2008. Disponível em: <http://www.scielo.br/ scielo.php?script=sci_arttext\&pid=S010029452008000300021 $\& \operatorname{lng}=$ en\&nrm=iso>. Acesso em 11 nov. 2011. doi: 10.1590/ S0100-29452008000300021.

INSTITUTO ADOLFO LUTZ. Normas analíticas do Instituto Adolfo Lutz: métodos químicos e físicos para análise dos alimentos. 3.ed. São Paulo: Instituto Adolfo Lutz, 1985. V.1, 371p.

INSTITUTO AGRONOMICO DO PARANÁ. Cartas climáticas. Disponível em: <http://www.iapar.br/modules/ conteudo/conteudo.php? conteudo=677>. Online. Acesso em: 20 out. 2010 a.
INSTITUTO AGRONOMICO DO PARANÁ. Médias históricas das estações do IAPAR. Disponível em: <http:// $200.201 .27 .14 / \mathrm{Site} / \mathrm{S} \mathrm{ma} / \mathrm{Estacoes}$ _IAPAR/ Estacoes_Parana.htm>. Online. Acesso em: 20 ago. 2010b.

JUBILEU, B.S. et al. Caracterização fenológica e produtiva das videiras 'Cabernet Sauvignon' e 'Alicante' (Vitis vinifera L.) produzidas fora de época no Norte do Paraná. Revista Brasileira de Fruticultura, Jaboticabal, v.32, n.2, p.451462, 2010. Disponível em: <http://www.scielo.br/ scielo.php?script=sci_arttext\&pid=S01002945201000020001 $5 \& \operatorname{lng}=$ en $\&$ nrm=iso>. Acesso em: 20 nov. 2010. doi: $10.1590 / \mathrm{S} 010029452010005000051$.

KISHINO, A.Y.; CARAMORI, P.H. Fatores climáticos e o desenvolvimento da videira. In: _____- et al. Viticultura tropical: o sistema de produção do Paraná. Londrina: Iapar, 2007. Cap3, p.59-86.

LEÃO, P.C.S. et al. Principais cultivares. In: SOARES, J.M.; LEÃO, P.C.S. A vitivinicultura no semiárido brasileiro. Brasília: Embrapa Informação Tecnológica; Petrolina: Embrapa Semiárido, 2009. Cap. 5, p.151-214.

ORLANDO, T.G.S. et al. Comportamento das cultivares Cabernet Sauvignon e Syrah em diferentes porta-enxertos. Ciência e Agrotecnologia, Lavras, v.32, n.3, p.749755, 2008. Disponível em: <http://www.scielo.br/ scielo.php? script $=$ sci_arttext\&pid $=S 141370542009000$ 300009>. Acesso em 20 nov. 2010. doi: 10.1590/S141370542009000300009 .

RIZZON, L.A. et al. Evolução da acidez durante a vinificação de uvas tintas de três regiões viticolas do Rio Grande do Sul. Ciências e Tecnologia de Alimentos, Campinas, v.18, n.2, p.179-183, 1998. Disponível em: <http://www.scielo.br/ scielo.php?pid=S010384782002000300023\&script=sci_arttext $>$. Acesso em: 20 nov. 2010. doi: 10.1590/S010384782002000300023 .

SOUZA, C.M. et al. Indicação de cultivares de videira para o Sul de Minas Gerais. In: REGINA, M.A. et al. Viticultura e enologia: atualizando conceitos. Andradas: EPAMIG, 2002. 340p.

WINKLER, A.J. et al. General viticulture. 2.ed. Berkeley: University of California, 1974. 710p.

WYLER, P. et al. Avaliação das características enológicas da cv Alicante Bouschet produzida em uma região tropical, no Nordeste do Brasil. In: CONGRESSO LATINO-AMERICANO DE VITICULTURA E ENOLOGIA, 11., 2007, Mendoza. Anais... Mendoza: Instituto Nacional de Vitivinicultura, 2007. CD. 